\title{
Atresia Esofágica y Fístula Traqueoesofágica. Evolución y Complicaciones Postquirúrgicas
}

\author{
LETICIA JAKUBSON S. ${ }^{1}$, FERNANDO PAZ C. ${ }^{2}$, ALEJANDRO ZAVALA B. ${ }^{3}$, \\ PAUL R. HARRIS D. ${ }^{4}$, PABLO BERTRAND N. ${ }^{5}$ \\ 1. Becada de Programa de Enfermedades Respiratorias del Niño, Hospital Exequiel González Cortés. Universidad de Chile. \\ 2. Pediatra Neumólogo, Hospital de Quillota. \\ 3. Cirugía Pediátrica, Hospital Clínico Pontificia Universidad Católica de Chile. \\ 4. Profesor Asociado, Facultad de Medicina, Universidad Católica de Chile. \\ 5. Profesor Asistente, Facultad de Medicina, Pontificia Universidad Católica de Chile. \\ Departamento de Pediatría, Sección de Respiratorio, Gastroenterología y Departamento de Cirugía, \\ Pontificia Universidad Católica de Chile.
}

\section{ABSTRACT \\ Esophageal Atresia and Tracheoesophagic Fistula. Evolution and Postsurgical Complications}

Esophageal atresia (EA) and tracheoesophageal fistula are congenital anomalies associated with respiratory and gastrointestinal morbidity. Objective: The aim of this study was to describe clinical characteristics of patients with EA and TEF during childhood, specially focused on respiratory and gastrointestinal complications. Methods: We reviewed the clinical charts from fifteen patients admitted to our hospital and who were followed locally between 1995 and 2007. Results: Gross classification distribution was: A: 0, B: 0, C: 12, D: 2, E: 1. Waterston distribution was A1: 6, B1: 1, B2: 4 y C2: 4. Associated malformations to EA and TEF were $7 / 15$. Main morbidity during the follow up period were: Recurrent pneumonia: $6 / 15$, recurrent wheezing $12 / 15$, tracheomalacia in $9 / 15$ and apnea in one patient. Fistula re-opening in 3/15, Gastroesophageal reflux in 15/15 esophagic stenosis in 7/15. All patients were admitted to the hospital at least once during the follow up and in 12/15 of this were due to respiratory causes. Conclusions: EA and TEF after surgical repair have significant respiratory and gastrointestinal morbidity and frequent admission to the hospital during the first years of life.

(Key words: Esophageal Atresia; Tracheoesophageal Fistula).

Rev Chil Pediatr 2010; 81 (4): 339-346

\section{RESUMEN}

La Atresia esofágica (AE) con o sin fístula traqueoesofágica (FTE) es una malformación congénita que se asocia a complicaciones respiratorias y digestivas. Objetivo: Describir las características de estos pacientes,

Trabajo recibido el 07 de agosto de 2009, devuelto para corregir el 22 de septiembre de 2009, segunda versión el 04 de junio de 2010, aceptado para publicación el 14 de junio de 2010.

Correspondencia a:

Dr. Pablo Bertrand N.

E-mail: bertrand@med.puc.cl 
su morbilidad respiratoria, digestiva y hospitalizaciones en su evolución a largo plazo. Materiales y Métodos: Se revisaron en forma retrospectiva 15 fichas de pacientes con diagnóstico de FTE evaluados por el equipo de Neumología Pediátrica de la Universidad Católica de Chile entre 1995 y 2007. Resultados: $\mathrm{N}=15$ pacientes, sexo masculino 9 diagnóstico el primer día de vida $13 / 15$ pacientes. Clasificación de Gross: $\mathrm{A}=0, \mathrm{~B}=0, \mathrm{C}=$ 12, $\mathrm{D}=2$, $\mathrm{E}=1$. Clasificación de Waterston A1: 6, B1: 1, B2: 4 y C2: 4 Malformaciones asociadas: en 7/15. Complicaciones respiratorias: Neumonía recurrente $8 / 15$, sibilancias recurrentes $12 / 15$, Recurrencia de fístula: 3 /15 pacientes, Apnea: 1 paciente, Traqueomalacia (TM) (9/15). Complicaciones digestivas: Reflujo gastroesofágico 15/15, Estenosis esofágica: 7/ 15. Un paciente requirió cirugía de Nissen. Todos los pacientes se hospitalizaron en al menos una oportunidad y en el 12/15 la causa fue respiratoria. Conclusión: La FTE es una enfermedad de resolución quirúrgica con alta morbilidad respiratoria, digestiva y frecuentes hospitalizaciones en los primeros años de vida.

(Palabras clave: Atresia Esofágica, fístula Traqueoesofágica).

Rev Chil Pediatr 2010; 81 (4): 339-346

\section{Introducción}

La Atresia Esofágica (AE), con o sin Fístula Traqueoesofágica (FTE), es una malformación congénita frecuente que afecta aproximadamente 1 de cada 3500 recién nacidos ${ }^{1,2}$. Esta malformación consiste en la interrupción de la continuidad del esófago que puede tener o no comunicación con la tráquea. En más del 50\% de los casos se asocia a otras malformaciones congénitas como Cardíacas (29\%), Genitourinarias $(14 \%)$, Anorectales (14\%) y Gastrointestinales $(13 \%)^{3,4}$. La AE y FTE forma parte de las asociaciones VACTERL y CHARGE y se encuentra asociada con frecuencia a otros síndromes genéticos como trisomía 21 y trisomía $18^{2,4}$; sin embargo, la mayoría de los casos de AE y FTE son esporádicos.

Los avances en cuidados intensivos en neonatología y en cirugía han permitido disminuir la mortalidad en niños con esta enfermedad cuya sobrevida actual es cercana al $90 \%$ tanto en el extranjero como en nuestro país ${ }^{5-7}$.

Aun cuando la mortalidad ha mejorado, la mayoría de los pacientes con AE y FTE presentan morbilidad significativa. Las complicaciones más frecuentes que presentan los pacientes con AE/FTE luego de la reparación quirúrgica son de tipo respiratoria y digestiva, que condicionan un deterioro de la calidad de vida y alta tasa de hospitalización ${ }^{8,9}$. Estos problemas suelen persistir aun después de realizada la cirugía correctora debido a múltiples factores: reflujo gastroesofágico, aspiración de contenido gástrico, inestabilidad de la vía aérea o traqueomalacia, epitelio anormal de la traquea, motilidad esofágica anormal, estenosis esofágica o recurrencia de la $\mathrm{FTE}^{1,10}$.

Aun cuando existen reportes en series extranjeras con buenos resultados ${ }^{7-12}$, la mayoría corresponde a niños operados hace dos décadas cuando la mortalidad a corto plazo era mucho mayor. En nuestro país la literatura es escasa $^{5,6,13}$ y reporta mejoría en la sobrevida general pero muestra poco detalle en la morbilidad asociada en esta enfermedad. El objetivo de este estudio fue describir las características generales de estos pacientes poniendo énfasis en su evolución y morbilidad asociada tanto respiratoria como digestiva durante los primeros años de vida.

\section{Material y Métodos}

Estudio descriptivo y retrospectivo en el cual se revisaron las fichas clínicas de un total de 18 pacientes con diagnóstico de AE y/o FTE que fueron evaluados en el Hospital Clínico de la Pontificia Universidad Católica de Chile, en un período comprendido entre enero de 1995 y junio de 2007. En 3 pacientes no se logró obtener información completa de modo que fueron excluidos. Se confeccionó un protocolo en el que se registraron variables demográficas y detalles significativos de la cirugía y sus complicaciones. Además se clasificaron los pacientes de acuerdo a las clasificación antató- 
mica de Gross y de pronóstico de Waterstone ${ }^{14}$ y Spitz ${ }^{15}$. En relación con las malformaciones o alteraciones cromosómicas asociadas se registró el tipo y número. Se registró el número de hospitalizaciones por causa respiratoria y digestiva. Los pacientes fueron evaluados 3 a 6 veces por año.

Con respecto a las complicaciones respiratorias se registró: neumonía recurrente (3 o más episodios de neumonía durante el seguimiento), traqueomalacia (visualización endoscópica de obstrucción traqueal mayor al 50\% del lumen en fase espiratoria), sibilancias recurrentes (3 o más episodios de obstrucción bronquial en el seguimiento), apnea (cese de la respiración observada por sus cuidadores). Se realizó una revisión crítica de los estudios de fibrobroncoscopías (FBC) realizadas en nuestra institución y se registraron los hallazgos. En 12 de 15 pacientes se realizó FBC por presentar enfermedades recurrentes como sibilancias o neumonías. Los reportes consignaron la presencia de traqueomalacia significativa.

Las complicaciones digestivas consideradas fueron: RGE (esofagograma, cintigrafía de RGE y/o phmetría de acuerdo a los hallazgos), estenosis esofágica (por medio de visualización endoscópica), número de dilataciones esofágicas, filtración de la anastomosis, perforación esofágica, obstrucción por cuerpo extraño y refistulización con el tratamiento realizado. El protocolo de evaluación de alteraciones digestivas se realizó en forma progresiva de acuerdo a su complejidad.

Los datos se describieron con mediana y rango o porcentaje. Para el análisis de las variables dicotómicas se utilizó el test de fisher y para las variables continuas el test de Kruskal Wallis. Se consideró una diferencia significativa la probabilidad $\mathrm{p}<0,05$.

\section{Resultados}

Se incluyen 15 pacientes, 9 varones, mediana de edad 6 años (rango 0,2-12 años). La mediana de edad gestacional fue 37 semanas (rango 34-40) y la mediana de peso y talla de nacimiento fueron $2665 \mathrm{~g}$ (rango 1 1503720 ) y $48 \mathrm{~cm}$ (rango 52-37).

En 13 pacientes el diagnóstico se realizó el primer día de vida. En un paciente el diagnóstico se hizo al segundo día de vida presentando AE con FTE proximal y distal. En un paciente el diagnóstico se realizó fuera del período neonatal y correspondió a un niño que presentó FTE tipo $\mathrm{H}$ cuyo estudio se confirmó a los 6 meses de vida. Doce de los 15 pacientes fueron operados en las primeras $48 \mathrm{hrs}$ de vida, de los 3 pacientes que se operaron después de las $48 \mathrm{hrs}$ de vida; uno fue al $4^{\circ}$ día coincidiendo con que su diagnóstico se hizo al $2^{\circ}$ día, y otro se operó el día 11 asociándose a su cirugía correctora colostomía y gastrostomía. Sólo un paciente fue operado fuera del período neonatal, lo que ocurrió al $7^{\circ}$ mes de vida cuando se realizó el diagnóstico.

El tipo de cirugía primaria fue mayoritariamente: cierre y anastomosis término-terminal (TT) que se realizó en $11(73,3 \%)$ pacientes, cierre con anastomosis TT y gastrostomía fue realizada en 1 paciente que presentó malformación asociada (VACTERL), gastrostomía y Esofagostomía se efectuó en 3 pacientes (estos pacientes presentaban malformaciones congénitas asociadas como S. Down, VACTERL $\mathrm{y}$ atresia anal) y un paciente con doble fístula requirió ascenso gástrico. Las malformaciones asociadas se encontraron en 8 de 15 pacientes $(53,3 \%)$, siendo más frecuentes las gastrointestinales $(30 \%)$. Los detalles de los pacientes se resumen en la Tabla 1.

Según la clasificación de Gross los pacientes se clasificaron: 12 niños con tipo $C, 2$ niños con tipo D y 1 niño con tipo E (figura 1). Con respecto a la clasificación de factores pronósticos de Spitz: 14/15 se los niños se encontraba en el grupo I, y un paciente se encontraba en el grupo II por presentar peso de nacimiento $<1500 \mathrm{~g}$ ).

Las complicaciones postquirúrgicas en el período agudo fueron reportadas en 5 pacientes (infecciosas 3, neumotórax 1, perforación esofágica 1 y estenosis subglótica 1). La duración de la hospitalización en los pacientes con complicaciones posquirúrgicas y sin estas fue similar. Se realizó fibrobroncoscopía a 12 de los 15 pacientes (rango de FBC por paciente 1-5) Se obtuvieron registros del procedimiento en 10 pacientes: traqueomalacia moderada 9 , variante anatómica del LM o LSD 2, bronquio 
Tabla 1. Características generales y morbilidad asociada de una serie de 15 pacientes con atresia esfofágica con fístula traqueoesofágica

\begin{tabular}{|c|c|c|c|c|c|c|}
\hline $\begin{array}{c}n= \\
15\end{array}$ & $\begin{array}{l}\text { Edad } \\
\text { actual }\end{array}$ & $\begin{array}{l}\text { Malformación/ } \\
\text { Asociación }\end{array}$ & Cirugía & $\begin{array}{l}\text { Complicaciones } \\
\text { respiratorias }\end{array}$ & $\begin{array}{c}\text { Complicaciones } \\
\text { digestivas }\end{array}$ & $\begin{array}{c}n \\
\text { Hosp. }\end{array}$ \\
\hline 1 & $3 \mathrm{~m}$ & $\begin{array}{l}\text { Hemivertebra } \\
\text { D. pielocalicial }\end{array}$ & $\begin{array}{l}\text { Cierre. } \\
\text { Anastomosis } \\
\mathrm{T}-\mathrm{T}\end{array}$ & Traqueomalacia & $\begin{array}{l}\text { RGE } \\
\text { Recurrencia de FTE }\end{array}$ & 1 \\
\hline 2 & 6 a $6 \mathrm{~m}$ & & $\begin{array}{l}\text { Cierre. } \\
\text { Anastomosis } \\
\text { T-T }\end{array}$ & $\begin{array}{l}\text { Neumonía recurrente } \\
\text { Sibilancias recurrentes }\end{array}$ & $\begin{array}{l}\text { RGE } \\
\text { Estenosis esofágica }\end{array}$ & 2 \\
\hline 3 & $9 a$ & Laringomalacia & $\begin{array}{l}\text { Cierre. } \\
\text { Anastomosis } \\
\text { T-T }\end{array}$ & $\begin{array}{l}\text { Traqueomalacia } \\
\text { Sibilancias recurrentes }\end{array}$ & $\begin{array}{l}\text { RGE Estenosis } \\
\text { esofágica. } \\
\text { Recurrencia de FTE }\end{array}$ & 2 \\
\hline 4 & $5 a$ & VACTERL & $\begin{array}{l}\text { Cierre. } \\
\text { Anastomosis } \\
\text { T-T } \\
\text { Gastrostomía. } \\
\text { Colostomía }\end{array}$ & $\begin{array}{l}\text { Neumonía recurrente } \\
\text { Sibilancias recurrentes }\end{array}$ & $\begin{array}{l}\text { RGE } \\
\text { Estenosis esofágica }\end{array}$ & 6 \\
\hline 5 & 5 a $3 \mathrm{~m}$ & Atresia anal & $\begin{array}{l}\text { Esofagostomía } \\
\text { Gastrostomía }\end{array}$ & Sibilancias recurrentes & RGE & 1 \\
\hline 6 & 7 a $10 \mathrm{~m}$ & $\begin{array}{l}\text { S. Down } \\
\text { Atresia duodenal }\end{array}$ & $\begin{array}{l}\text { Esofagostomía } \\
\text { Gastrostomía }\end{array}$ & $\begin{array}{l}\text { Traqueomalacia. Apnea } \\
\text { Sibilancias recurrentes }\end{array}$ & $\begin{array}{l}\text { RGE } \\
\text { Estenosis esofágica }\end{array}$ & 4 \\
\hline 7 & $12 \mathrm{a}$ & & $\begin{array}{l}\text { Cierre. } \\
\text { Anastomosis } \\
\text { T-T }\end{array}$ & $\begin{array}{l}\text { Neumonía recurrente } \\
\text { Sibilancias recurrentes }\end{array}$ & $\begin{array}{l}\text { RGE } \\
\text { Trastorno deglución }\end{array}$ & 6 \\
\hline 8 & 9 a $2 \mathrm{~m}$ & Ano imperforado & $\begin{array}{l}\text { Cierre. } \\
\text { Anastomosis } \\
\text { T-T }\end{array}$ & $\begin{array}{l}\text { Traqueomalacia } \\
\text { Sibilancias recurrentes }\end{array}$ & $\begin{array}{l}\text { RGE } \\
\text { Estenosis esofágica }\end{array}$ & 2 \\
\hline 9 & $7 a$ & & $\begin{array}{l}\text { Cierre. } \\
\text { Anastomosis } \\
\text { T-T }\end{array}$ & $\begin{array}{l}\text { Neumonía recurrente } \\
\text { Traqueomalacia } \\
\text { Sibilancias recurrentes }\end{array}$ & $\begin{array}{l}\text { RGE } \\
\text { Estenosis esofágica } \\
\text { Trastorno deglución }\end{array}$ & 3 \\
\hline 10 & 6 a $1 \mathrm{~m}$ & $\begin{array}{l}\text { Coronaria } \\
\text { anómala }\end{array}$ & $\begin{array}{l}\text { Cierre. } \\
\text { Anastomosis } \\
\text { T-T }\end{array}$ & $\begin{array}{l}\text { Neumonía recurrente } \\
\text { Traqueomalacia } \\
\text { Sibilancias recurrentes }\end{array}$ & RGE & 10 \\
\hline 11 & $2 a$ & & $\begin{array}{l}\text { Sección y } \\
\text { cierre }\end{array}$ & Sibilancias recurrentes & RGE & 1 \\
\hline 12 & 7 a $6 \mathrm{~m}$ & & $\begin{array}{l}\text { Cierre. } \\
\text { Anastomosis } \\
\text { T-T }\end{array}$ & $\begin{array}{l}\text { Neumonía recurrente } \\
\text { Traqueomalacia } \\
\text { Sibilancias recurrentes }\end{array}$ & RGE & 1 \\
\hline 13 & 4 a $7 \mathrm{~m}$ & & $\begin{array}{l}\text { Cierre. } \\
\text { Anastomosis } \\
\text { T-T }\end{array}$ & $\begin{array}{l}\text { Neumonía recurrente } \\
\text { Traqueomalacia } \\
\text { Sibilancias recurrentes }\end{array}$ & RGE & 3 \\
\hline 14 & $2 a$ & & $\begin{array}{l}\text { Cierre. } \\
\text { Anastomosis } \\
\text { T-T }\end{array}$ & Traqueomalacia & $\begin{array}{l}\text { RGE. Estenosis } \\
\text { esofágica } \\
\text { Recurrencia de FTE }\end{array}$ & 2 \\
\hline 15 & $4 a$ & $\begin{array}{l}\text { VACTERL. } \\
\text { Bronquio carinal }\end{array}$ & $\begin{array}{l}\text { Esofagostomía } \\
\text { Gastrostomía } \\
\text { Ascenso gas- } \\
\text { trico }\end{array}$ & $\begin{array}{l}\text { Neumonía recurrente } \\
\text { Traqueomalacia } \\
\text { Sibilancias recurrentes }\end{array}$ & $\begin{array}{l}\text { RGE } \\
\text { Estenosis esofágica } \\
\text { Trastorno deglución }\end{array}$ & 14 \\
\hline
\end{tabular}

VACTERL: Defectos vertebrales, malformaciones anorrectales, defectos cardíacos, Atresia esofágica y Fístula traqueoesofágica, alteraciones renales y de vía urinaria, anomalías de los miembros. D. pielocalicial: Dilatación pielocalicial. T-T: Término terminal. RGE: Reflujo gastroesofágico. Edad: a (años), m (meses). 


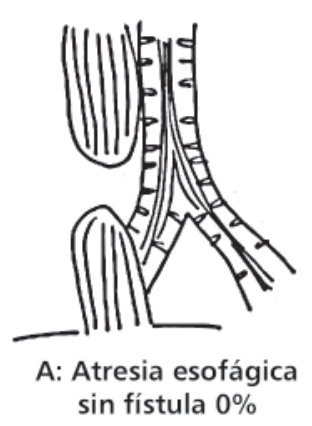

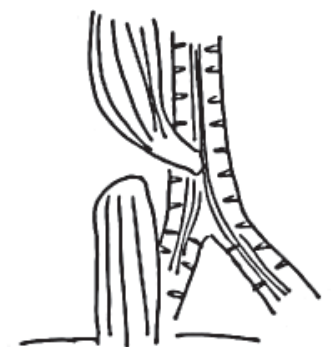

B: Atresia esofágica con fístula proximal $0 \%$

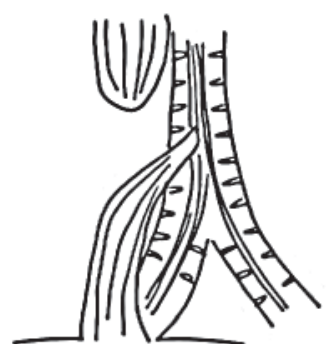

C: Atresia esofágica con fístula distal $80 \%(12)$

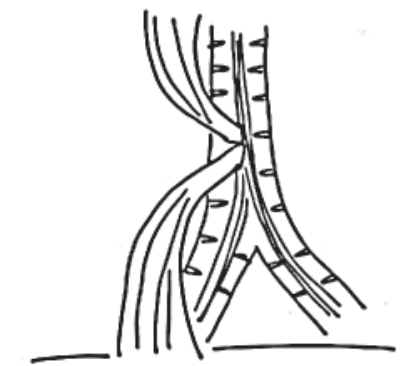

D: Atresia esofágica con fístula proximal y distal $13 \%$ (2)

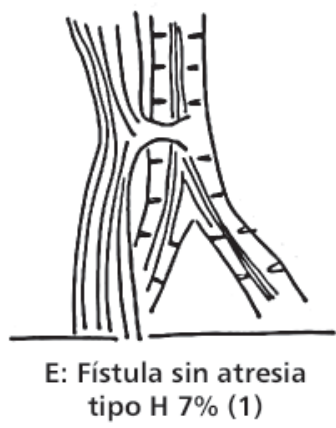

tipo $\mathrm{H} \mathrm{7 \%} \mathrm{(1)}$
Figura 1. Clasificación de Gross. accesorio y carinal 2, granuloma de cuerdas vocales 1 , laringomalacia 1 .

El seguimiento se realizó por una mediana de 4 años (0-7). En este período se reportó la aparición de sibilancias en 12/15 y neumonía recurrente en $8 / 15$ pacientes. Se comparó a los pacientes con y sin malformaciones con respecto a la frecuencia de neumonías y hospitalizaciones, no encontrando diferencias significativas.

Todos los pacientes presentaron reflujo gastroesofágico (RGE) de alguna magnitud de acuerdo al algoritmo de estudio mencionado. Sólo 1 paciente requirió cirugía de Nissen como tratamiento de RGE. Estenosis esofágica se presentó en 7 de 15 pacientes y la totalidad de estos niños fue tratado con dilataciones esofágicas con un rango de 1-4 dilataciones por paciente. En un paciente se reportó extracción endoscópica de cuerpo extraño esofágico.

La recurrencia de FTE se presento en 3 de15 pacientes. En 2 de ellos se realizó cierre endoscópico con Beriplast. En uno la conducta fue expectante hasta el momento que se realizó este reporte. Características de la serie y su morbilidad asociada se muestran en la tabla 1 .
Todos los pacientes requirieron hospitalizarse, lo que ocurrió con una mediana de 2 veces en el período de seguimiento (rango 1-8). La mayoría de los pacientes (12/15) se hospitalizó por causa respiratoria. Se comparó el número de hospitalizaciones de los pacientes con y sin malformaciones asociadas y de los pacientes con y sin complicaciones posquirúrgicas inmediatas, no encontrando diferencia significativa en dichos grupos. No hubo mortalidad en esta serie.

\section{Discusión}

El presente estudio describe la evolución de los primeros años de vida de una serie de pacientes con AE y FTE, que desarrollaron múltiples complicaciones respiratorias y digestivas. Esta pequeña serie aporta con nueva evidencia que cuantifica la morbilidad asociada y las consecuentes hospitalizaciones. Estos hallazgos deben ser difundidos en nuestro medio con el objeto de anticipar la morbilidad mencionada.

El pronóstico de AE y FTE ha mejorado mucho en los últimos años, siendo la sobrevida 
actual mayor al 95\% en centros de derivación con cuidados intensivos neonatales ${ }^{16}$. En nuestro país la sobrevida ha mejorado en forma categórica conforme han pasado los años ${ }^{5,6,13}$ lo que se proyecta aún más en nuestra serie. Usando como comparador la clasificación de Waterston ${ }^{13}$ la sobrevida de nuestros pacientes fue de $100 \%$. Recientemente, se ha validado el uso de la clasificación de Spitz ${ }^{14}$ que de forma más precisa podría valorar el riesgo vital que presentan los pacientes considerando sólo peso de nacimiento y presencia de malformaciones cardíacas como predictores. En este estudio, López ${ }^{17}$ reporta sobrevidas por grupo de I: $98,5 \%$, II: $82 \%$ y III: $50 \%$ Okamoto $^{18}$, cuestiona esta clasificación y postula una nueva fórmula que podría ser mejor predictor de mortalidad agregando un grupo de mayor riesgo. Nuestros resultados aparecen representados en el grupo de menor riesgo con sobrevida similar a lo reportado en la literatura internacional.

Si se analiza la distribución de AE y FTE de acuerdo a la Clasificación Anatómica de Gross, la malformación denominada tipo C (AE más FTE distal) fue la más frecuente lo que concuerda con datos publicados en Chile e internacionalmente ${ }^{2,3,5,11-13}$, asimismo con el momento del diagnóstico y edad de cirugía ${ }^{1-13}$.

La AE y FTE se presenta frecuentemente en asociación con malformaciones congénitas y síndromes genéticos. Las cifras reportadas en la literatura nacional e internacional son variables y dependen fundamentalmente del número de pacientes y del tipo de población. Nuestros resultados fueron similares a lo reportado en series más grandes ${ }^{18}$.

La morbilidad respiratoria y digestiva que presentan pacientes con AE y FTE ha sido reportada previamente ${ }^{1,8}$. En esta pequeña serie es muy evidente la frecuencia de complicaciones en el seguimiento de los primeros años de vida. Muchos factores pueden contribuir a la recurrencia de síntomas respiratorios. Algunos autores ${ }^{19}$ sugieren que la presencia de epitelio escamoso no ciliar en la tráquea podría empeorar seriamente el mecanismo de barrido ciliar, siendo éste un importante mecanismo de defensa del tracto respiratorio que predispone a la aparición de atelectasias y neumonías recu- rrentes. También existe una asociación clara entre la severidad del RGE y la persistencia de los síntomas, que en nuestra serie afectó a la totalidad de los niños. La estenosis esofágica y la alteración del peristaltismo esofágico puede generar acumulación de restos de comida en el tercio superior del esófago y así llegar a la tráquea causando episodios de aspiración ${ }^{1,9,10}$. Chetcuti ${ }^{9}$ reporta sibilancias en el $63 \%$ de los niños menores de 5 años, bronquitis en el $74 \%$ y neumonías en el $35 \%$ de los niños con $\mathrm{AE}$ y FTE. Nuestra cifra de sibilancias encontradas es superior aunque incluye probablemente a aquellos pacientes que este autor define como bronquitis. En nuestros pacientes la alta prevalencia de sibilancias se explica en parte por la frecuente presencia de traqueomalacia. Estudios extranjeros describen que las infecciones respiratorias recurrentes ocurren más frecuentemente en los primeros 5 años de vida y tienden a mejorar con la edad. Debido a que nuestro seguimiento involucra principalmente los primeros años de vida creemos que estos datos corroboran la experiencia internacional. Sobretodo cuando existe evidencia que estos pacientes no presentan marcadores inflamatorios ni evidencia de remodelación, lo que hace menos probable el diagnóstico de asma ${ }^{20}$. Este dato es muy gravitante puesto que permite evitar tratamientos ineficaces y prolongados que no cuentan con justificación alguna.

Traqueomalacia está presente en el 75\% de las muestras de anatomía patológica de los pacientes con AE y/o FTE, pero se reporta como clínicamente significativa en el $20 \%$ de los pacientes con esta enfermedad. Su aparición se explica por la alteración primaria de la estructura traqueal que compromete en forma variable su estabilidad. De nuestros pacientes, 12 fueron estudiados con fibrobroncoscopías y 9 presentan traqueomalacia lo que es muy similar a los reportado por otros autores con cifras de $60 \%{ }^{20}$ y $78 \%{ }^{21}$.

Muchos de los pacientes con Traqueomalacia reciben broncodilatadores beta agonistas, los que podrían relajar aun más el tono del músculo liso de la pared traqueal, empeorando los síntomas. La TM tiende a mejorar con la edad, debido al crecimiento de la vía aérea que hace más rígida su estructura y por tanto 
disminuye el colapso dinámico.

Muchas series reportan reflujo gastroesofágico (RGE) en el 50\% de los pacientes, aunque está universalmente presente en algún grado en todos los pacientes ${ }^{22}$. En nuestra serie, la totalidad de los pacientes presentaron RGE, que creemos es atribuible a una mayor sensibilidad de los tratantes en su búsqueda. Al igual que la maduración que sufre la tráquea, el esófago va creciendo y su evolución natural es hacia la mejoría con la edad. Sólo la minoría de los pacientes requiere cirugía.

Así como ocurre que los pacientes con $\mathrm{AE}$ y FTE presentan mayor morbilidad durante los primeros cinco años de vida, asimismo también presentan una más alta tasa de hospitalización en este período. Un estudio en Australia reporta que el $44 \%$ de los pacientes se hospitaliza por causa respiratoria, y la mayoría se producen en los primeros 5 años de vida. Esta realidad se repite en nuestros resultados. Los niños con FTE presentan una susceptibilidad mayor a infecciones virales comunes, de modo que los episodios de sibilancias recurrentes son frecuentes. Esto explica las hospitalizaciones y la mayor frecuencia de sibilancias e infecciones respiratorias recurrentes que se describen en la literatura y que muestra nuestra serie. Es por esto que es importante anticiparse, educar y asesorar a la familia, mantener calendario inmunizaciones al día y realizar un seguimiento multidisciplinario.

Este estudio retrospectivo y descriptivo revela la importante morbilidad respiratoria y digestiva que aparece en el seguimiento a largo plazo en pacientes con AE y FTE. Es importante dar a conocer esta realidad para anticipar el desafío clínico que estos pacientes plantean. Asimismo se hace indispensable complementar esta información con estudios de evolución clínica a más largo plazo.

\section{Referencias}

1.- Kovesi T, Rubin S: Long- term Complications of Congenital Esophageal Atresia and/or Tracheoesophageal Fistula. Chest 2004; 126: 915-25.

2.- Spitz L: Oesophageal atresia. Orphanet Journal of Rare Diseases 2007; 2: 24.
3.- Robert E, Mutchinick O, Mastroiacovo P, et al: An international collaborative study of the epidemiology of esophageal atresia or Stenosis. Reprod Toxicol 1993; 7 : 405-21.

4.- Chittmittrapap S, Sptiz L, Kiely EM, Brereton RJ: Oesophageal atresia and associated anomalies. Arch Dis Child 1989; 64: 364-8.

5.- Fierro C, Caro M, Anzieta J, Butte J, González P, Apablaza J: Atresia esofágica. Manejo quirúrgico en el Hospital clínico regional de Valdivia. Cuad Cir 2002; 16: 20-5.

6.- Weldt E, Caamaño E, Escobar J, et al: Atresia esofágica, análisis y seguimiento. Rev Chil Pediatr 1998; 69: 241-6.

7.- Konkin D, O'Hali W, Webber E, Blair G: Outcomes in Esophageal Atresia and Tracheoesophageal Fistula. J Pediatr Surg 2003; 38: 1726-9.

8.- Little D, Rescorla F, Grosfeld J, West K, Scherer L, Engum S: Long-Term Analysis of Children Whith Esophageal Atresia and Tracheoesophageal Fistula. J Pediatr Surg 2003; 38: 852-6.

9.- Chetcuti P, Phelan P: Respiratory morbidity after repair of esophageal atresia and tracheo-oesophageal fistula. Arch Dis Child 1993; 68: 167-70.

10.- Agrawal L, Beardsmore C, MacFadyen U: Respiratory function in childhood following repair of esophageal atresia and tracheoesophageal fistula. Arch Dis Child 1999; 81: 404-8.

11.- Teich S, Barton DP, Ginn-Pease M, King D: Prognostic classification for Esophageal Atresia and Tracheoesophageal Fistula: Waterston versus Montreal. J Pediatr Surg 1997; 32: 1075-9.

12.- Sompii E, Tammela O, Ruuska T: Outcome of Patients Operated on for Esophageal Atresia:30 Years' Experience. J Pediatr Surg 1998; 33: 1341-6.

13.- Ossandón F, Acuña R, Sirebrenik S, Krebs C, Bravo I: Atresia esofágica. Evaluación, manejo y seguimiento de 18 casos. Rev Chil Pediatr 1992; 63: 84-8.

14.- Waterston DJ, Bonham Carter RE, Aberdeen E: Esophageal atresia: Tracheo-esophageal fistula. A study of survival in 218 infants. Lancet 1962: 819-22.

15.- Spitz L, Kiely EM, Morecroft JA, et al: Oesophageal atresia: At risk groups for the 1900s. J Pediatr Surg 1994; 29: 723-5.

16.- Gupta DK, Sharma S: Esophageal atresia: the total care in high-risk population. Semin Pediatr Surg 2008; 17 : 236-43.

17- López PJ, Keys C, Pierro A, et al: Oesophageal atresia: improved outcome in high-risk groups? J Pediatr Surg 2006; 41 (2): 331-4. 
18.- Okamoto T, Takamizawa S, Arai H, et al: Esophageal atresia: Prognostic classification revisited. Surgery. 2009; 145: 675-81.

19.- Emery JL, Haddadin AJ: Squamous epithelium in respiratory tract of children with trachea-oesophageal fistula. Arch Dis Child 1971; 46: 236-42.

20.- Malmström K, Lohi J, Lindahl H, Pelkonen A: Longitudinal follow-up of bronchial inflammation, respiratory symptoms, and pulmonary function in adolescents after repair of esophageal atresia with tracheoesophageal fistula. J Pediatr 2008; 153: 396-401.

21.- Briganti V, Oriolo L, Buffa V, Garofalo S, Cavallaro $S$, Calistia A: Tracheomalacia in oesophageal atresia: morphological considerations by endoscopic and CT study. Eur J Cardiothorac Surg 2005; 28: 11-5.

22.- Koivusalo A, Pakarinen M, Rintala R: The cumulative incidence of significant gastrooesophageal reflux in patients with oesophageal atresia with a distal fistula-a systematic clinical, $\mathrm{pH}$-metric, and endoscopic follow-up study. J Pediatr Surg 2007; 42: 370-4. 